\title{
Residual lesions in postoperative pediatric cardiac surgery patients receiving extracorporeal membrane oxygenation support
}

\author{
Hemant S. Agarwal, MBBS, FAAP, ${ }^{a}$ Daphne C. Hardison, VPNPP, ${ }^{b}$ Benjamin R. Saville, PhD, ${ }^{c}$ \\ Brian S. Donahue, MD, PhD, ${ }^{\mathrm{a}, \mathrm{d}}$ Fred S. Lamb, MD, PhD, ${ }^{\mathrm{a}}$ David P. Bichell, MD, ${ }^{\mathrm{e}}$ and Zena L. Harris, $\mathrm{MD}^{\mathrm{a}}$
}

Objective: The objective of this study was to examine the incidence and clinical outcomes of residual lesions in postoperative pediatric cardiac surgery patients receiving extracorporeal membrane oxygenation (ECMO) support.

\begin{abstract}
Methods: A retrospective observational study was undertaken at a pediatric heart institution. Postoperative pediatric cardiac surgery patients receiving ECMO support within 7 days of surgery during the past 7 years (2005-2011) were studied. A hemodynamically significant cardiac lesion on ECMO support that required intervention to decannulate successfully was defined as a residual lesion. Demographic data, complexity of cardiac defect, surgical data, indications for ECMO, echocardiographic findings, and cardiac catheterization results were studied. Evaluation of residual lesions based on duration of ECMO support, interventions undertaken, and clinical outcomes were also examined.
\end{abstract}

Results: Residual lesions were evaluated in 43 of 119 postoperative patients placed on ECMO support. Lesions were detected in 35 patients $(28 \%)$, predominantly in branch pulmonary arteries $(\mathrm{n}=10)$, shunts $(\mathrm{n}=7)$, and ventricular outflow tracts $(\mathrm{n}=9)$. Echocardiography detected 7 residual lesions $(20 \%)$ and cardiac catheterization detected 28 residual lesions $(80 \%)$. Earlier detection of residual lesions during the first 3 days of ECMO support in 24 patients improved their rate of decannulation significantly $(P=.004)$ and survival to hospital discharge $(P=.035)$, compared with later detection (after 3 days of ECMO support) in 11 patients.

Conclusions: Residual lesions are present in approximately one-quarter of postoperative cardiac surgery patients requiring ECMO support. All postoperative pediatric cardiac surgery patients unable to be weaned off ECMO successfully should be evaluated actively for residual lesions, preferably by cardiac catheterization imaging. Earlier detection of residual lesions and reintervention are associated with improved clinical outcome. (J Thorac Cardiovasc Surg 2014;147:434-41)

The first application of extracorporeal membrane oxygenation (ECMO) following palliative repair of congenital heart disease in pediatrics was reported in $1970 .{ }^{1}$ The use of ECMO in pediatric cardiac surgery has increased steadily during the past 3 decades. $^{2}$ The indications for ECMO support have broadened during this time to include low cardiac output, failure to wean from cardiopulmonary bypass, unexpected cardiac arrest, severe cyanosis, or refractory arrhythmias. ${ }^{3}$ The increase in ECMO use has been associated concomitantly with advances in oxygenator technology, enhanced coating material of the ECMO circuit internal surface, improved anticoagulation strategies, and increased

\footnotetext{
From the Department of Pediatrics, ${ }^{\mathrm{a}}$ ECMO Division, ${ }^{\mathrm{b}}$ Department of Pediatrics, and Department of Cardio-Thoracic Surgery, ${ }^{\mathrm{e}}$ Monroe Carell Jr. Children's Hospital at Vanderbilt, Nashville, Tenn; and Division of Pediatric Cardiac Anesthesia, ${ }^{c}$ Department of Biostatistics, and Department of Anesthesia, ${ }^{\mathrm{d}}$ Vanderbilt University Medical Center, Nashville, Tenn.

Disclosures: Authors have nothing to disclose with regard to commercial support. Received for publication Sept 10, 2012; revisions received Feb 15, 2013; accepted for publication March 15, 2013; available ahead of print April 18, 2013.

Address for reprints: Hemant S. Agarwal, MBBS, FAAP, 5121 Doctor's Office Tower, 2200 Children's Way, Nashville, TN 37232-9075 (E-mail: Hemant.Agarwal@ Vanderbilt.edu).

0022-5223/\$36.00

Copyright (C) 2014 by The American Association for Thoracic Surgery http://dx.doi.org/10.1016/j.jtcvs.2013.03.021
}

sophistication in blood flow controller use in the ECMO circuit. ${ }^{4}$ However, the mortality in pediatric cardiac surgery patients requiring ECMO support continues to remain high despite increased efficacy in ECMO circuits and postoperative cardiac care. ${ }^{2,4}$

Extracorporeal membrane oxygenation is a support modality and not a treatment by itself. The primary aim of ECMO support is to unload the heart and to decrease its work to allow complete recovery of cardiac function from surgery within a short period of time. Residual lesions in cardiac surgery patients receiving ECMO support ${ }^{5-8}$ may result in incomplete recovery of cardiac function. The clinical decision to study residual lesions in patients on ECMO support by means of echocardiography or cardiac catheterization is quite subjective, and is based largely on clinical judgment. ${ }^{5}$ Because there are few reports that have evaluated residual lesions and their outcomes in pediatric cardiac surgery patients placed on ECMO,,9 the primary aim of our study was to evaluate the incidence of hemodynamically significant residual lesions that required reintervention to separate successfully from ECMO support. Our secondary aim was to study clinical outcomes based on time period of residual lesion detection on ECMO support. 


\section{Abbreviations and Acronyms \\ $\mathrm{ECMO}=$ extracorporeal membrane oxygenation \\ $\mathrm{E}-\mathrm{CPR}=$ extracorporeal cardiopulmonary resuscitation}

\section{MATERIALS AND METHODS}

This single-institution retrospective study was undertaken at the Pediatric Heart Institute at the Monroe Carell Jr. Children's Hospital at Vanderbilt. The institution review board reviewed and approved the study protocol.

All consecutive postoperative pediatric ( $<18$ years) cardiac surgery patients placed on ECMO between January 1, 2005, and December 31, 2011, were included in our study. The postoperative period was defined as the time interval from cardiac surgery to postoperative day $7 .{ }^{10}$ Demographic data including age, weight, gender, primary cardiac defect, cardiac surgery undertaken, cardiopulmonary bypass time, and crossclamp time were studied. The severity of underlying disease and the procedure performed were assessed using the Risk Adjustment in Congenital Heart Surgery version 1 classification. ${ }^{11}$ Electronic medical records, echocardiographic findings, cardiac catheterization reports, and operative reports were examined for collection of our data.

Indications for ECMO support were subcategorized as failure to separate from cardiopulmonary bypass, low cardiac output status despite maximal isotropic and ventilatory support, cardiac arrest with ECMO use for cardiopulmonary resuscitation (extracorporeal cardiopulmonary resuscitation [E-CPR]), and others, including respiratory failure, pulmonary hypertension, or arrhythmias. ${ }^{3,7}$ The time interval from cardiac surgery to placement on ECMO, duration of ECMO support, and the presence of renal failure on ECMO as defined by a serum creatinine value $>1.5 \mathrm{mg} / \mathrm{dL}$ or the need for continuous renal replacement therapy on ECMO were recorded. Weaning and separation from ECMO support was based on either adequate cardiopulmonary stability, or the development of one or more incidences of irreversible organ dysfunction precluding survival. Any patient who was unable to tolerate successfully weaning of ECMO flows or a clamping trial of the ECMO circuit without significant escalation of inotropic support, volume resuscitation, and/or high ventilator requirements was considered to have failed the weaning trial of ECMO. Patients who failed the weaning trial of ECMO without a clear cause were investigated for presence of residual lesions. Imaging studies including transthoracic echocardiography and cardiac catheterization were undertaken to evaluate residual lesions. A transthoracic echocardiography imaging study was undertaken during the weaning process of ECMO flows and was, in general, the initial imaging study for evaluating residual lesions. A cardiac catheterization imaging study was undertaken when the echocardiographic results were inconclusive and patient was unable to be weaned off ECMO support successfully. Any hemodynamically significant cardiac defect that necessitated reintervention to separate successfully from ECMO was defined as a residual lesion. ${ }^{5}$ The recognition of residual lesions based on duration of ECMO support was evaluated. Residual lesions detected during the first 3 days of ECMO were labeled as early diagnosis; residual lesions detected after 3 days of ECMO support were labeled as late diagnosis. Decannulation from ECMO and survival to hospital discharge was studied for all patients.

Continuous variables are presented as median values and interquartile ranges, and categorical variables are presented as numbers and percentages in the tables and the text. To compare data between groups, we used 2-sided tests. The Wilcoxon rank sum test was used to compare continuous variables and Pearson's $\chi^{2}$ test was used to compare categorical variables. Yates correction was applied when appropriate. A $P$ value of .05 or less was considered to be significant.

\section{RESULTS}

During the 7-year study period, 3447 pediatric patients underwent cardiac surgery in our institution. One hundred ninety-two patients of 3447 patients $(5.5 \%)$ were placed on ECMO for cardiac indications. One hundred nineteen of 192 patients $(62 \%)$ were placed on ECMO postoperatively within the first 7 days of surgery. Extracorporeal membrane oxygenation during the postoperative period in these 119 patients was performed by central cannulation via the open chest.

The demographics, complexity of cardiac defect, cardiac surgery details, and ECMO details are shown in Table 1. Of the 119 postoperative patients placed on ECMO, 50 patients $(42 \%)$ were placed on ECMO coming off cardiopulmonary bypass, 25 patients $(21 \%)$ were placed on ECMO for low cardiac output status in the intensive care unit with elevated serum lactate levels and high inotrope and ventilator support, 34 patients $(29 \%)$ were placed on ECMO for E-CPR, and 10 patients $(8 \%)$ were placed for other reasons, including acute respiratory failure $(\mathrm{n}=5)$, pulmonary hypertension $(\mathrm{n}=3)$, and refractory arrhythmias $(\mathrm{n}=2)$. Thirty-four patients were placed on ECMO as E-CPR with a mean duration of $33 \pm 15.6$ minutes of conventional cardiopulmonary resuscitation before placement on ECMO. Seventy-five of 119 postoperative patients $(63 \%)$ placed on ECMO were decannulated successfully and 49 of these patients $(41 \%)$ survived to hospital discharge.

Forty-three of the 119 patients were evaluated for residual lesions by imaging studies because they were unable to be weaned successfully from ECMO support. Transthoracic echocardiographic imaging studies during the weaning process of ECMO flows were undertaken in 40 patients and cardiac catheterization imaging was undertaken in 36 patients to evaluate residual lesions. Transthoracic echocardiography identified residual lesions in 7 of the 40 patients $(17 \%)$ studied; cardiac catheterization identified residual lesions in 28 of the 36 patients ( $78 \%$ ) that underwent this study. Transthoracic echocardiography was undertaken in $59(78 \%)$ of the remaining 76 patients during the course of ECMO support. Echocardiography, however, was not done during the weaning process of ECMO flows in these patients, and in none of these 59 patients were any residual lesions detected. One of the remaining $76 \mathrm{pa}-$ tients underwent a cardiac catheterization study that revealed elevated end diastolic pressures in the right ventricle.

Residual lesions requiring reintervention were detected in 35 of the 43 patients $(81 \%)$ studied. The 7 residual lesions detected by transthoracic echocardiography included proximal branch pulmonary artery stenosis $(\mathrm{n}=3)$, Blalock-Taussig shunt thrombosis $(\mathrm{n}=2)$, patent ductus arteriosus $(\mathrm{n}=1)$, and pulmonary overcirculation secondary to a large Blalock-Taussig shunt $(\mathrm{n}=1)$. Transthoracic echocardiography was the only imaging study undertaken 
TABLE 1. Demographic and ECMO details of postoperative patients placed on ECMO within 7 days of surgery $(n=119)$

\begin{tabular}{lc}
\hline \multicolumn{1}{c}{ Variable } & Total (n = 119) \\
\hline Age, days; mean (IQR) & $12(6-79)$ \\
Weight, kg; mean (IQR) & $3.3(2.7-4.1)$ \\
Gender, n (\%) & \\
Male & $67(56)$ \\
Female & $52(44)$ \\
Ventricle physiology, n (\%) & \\
One & $58(49)$ \\
Two & $61(51)$ \\
RACHS, category; n (\%) & \\
2 & $11(9)$ \\
3 & $46(38)$ \\
4 & $15(13)$ \\
$5-6$ & $40(34)$ \\
Could not be ranked & $7(6)$ \\
CPB, min; mean (IQR) & $185(128-282)$ \\
ECMO indication, n (\%)* & \\
1 & $50(42)$ \\
2 & $25(21)$ \\
3 & $34(29)$ \\
4 & $10(8)$ \\
ECMO location, n (\%) & \\
Operating room & $58(49)$ \\
ICU & $61(51)$ \\
Surgery-ECMO interval, days; mean (IQR) & $0(0-0)$ \\
ECMO duration, days; mean (IQR) & $4(2-7)$ \\
Renal failure, n (\%) & $41(34)$ \\
Decannulation, n (\%) & $75(63)$ \\
Survival to hospital discharge, n (\%) & $49(41)$ \\
\hline$I Q R$, Interquartile range; RACHS, Risk Adjustment in Congenital Heart Surgery, \\
$C P B$, cardiopulmonary bypass; ECMO, Extracorporeal membrane oxygenation; \\
ICU, intensive care unit. *CMO indication: 1, off CPB; 2, low cardiac output; 3, car- \\
diac arrest requiring cardiopulmonary resuscitation; 4, other, including respiratory \\
failure, pulmonary hypertension, and arrhythmias. \\
$\quad$ \\
\hline
\end{tabular}

in these 7 patients to identify residual lesions. The 28 residual lesions detected by cardiac catheterization included branch pulmonary arteries stenosis $(n=7)$, right ventricular outflow tract stenosis $(\mathrm{n}=4)$, Blalock-Taussig or Sano shunt stenosis $(n=4)$, stenosis of aorta $(n=4)$, pulmonary vein stenosis $(\mathrm{n}=3)$, thrombus in aorta $(\mathrm{n}=2)$, pulmonary overcirculation $(\mathrm{n}=1)$, aortopulmonary collateral $(\mathrm{n}=1)$, mitral valve regurgitation $(n=1)$, and tricuspid valve regurgitation $(\mathrm{n}=1)$. Twenty-five of these 28 patients underwent transthoracic echocardiography during the weaning process of ECMO flows. There was some agreement of echocardiographic findings with cardiac catheterization results in 3 of these 25 patients for suspicion of residual lesions. Transthoracic echocardiography revealed moderate tricuspid regurgitation during the clamp trial of ECMO in 1 patient who had severe tricuspid regurgitation on cardiac catheterization that required reintervention, it revealed mild right ventricleto-pulmonary artery conduit stenosis in a second patient who had severe distal conduit stenosis and proximal branch pulmonary artery stenosis that required reintervention, and it revealed decreased blood flow in bilateral branch pulmonary arteries in the third patient who had diffuse branch pulmonary artery hypoplasia that required reintervention. Eight of the remaining 36 patients evaluated by cardiac catheterization revealed severe diastolic dysfunction $(\mathrm{n}=4)$, diffuse coronary artery thrombosis $(\mathrm{n}=2)$, and no hemodynamically significant residual lesions $(\mathrm{n}=2)$. The 2 patients in whom no residual lesions were detected had respiratory causes for their deterioration: viral pneumonia and multilobe atelectasis. All of these 8 patients underwent a transthoracic echocardiography imaging study during the weaning process of ECMO flow that was inconclusive. The types of residual lesions detected in our study based on the time period of ECMO support are shown in Table 2. Thirty-three of 43 patients $(77 \%)$ evaluated for residual lesions on ECMO in our study underwent a transthoracic or epicardial echocardiographic study at the end of their surgery.

Seven interventions for residual lesions were undertaken in the cardiac catheterization laboratory, 27 in the operating room, and 1 in the intensive care unit (Table 3). After the hemodynamically significant residual lesions were identified, they were corrected within the next 24 hours. The residual lesions that were identified in the cardiac catheterization laboratory and that were amenable to intervention in the laboratory were corrected at the time of the study, after discussion with the surgeon and cardiology team. If the lesions required surgical intervention, they were corrected within the next 24-hour period, depending on the earliest availability of the surgeon. There were no major complications during and after cardiac catheterization on ECMO, such as excessive blood loss requiring reexploration,

TABLE 2. Detection of residual lesions by echocardiography and/or cardiac catheterization in postoperative cardiac surgery patients placed on ECMO

\begin{tabular}{lc}
\hline \multicolumn{1}{c}{ Residual lesions } & $\mathbf{n}$ \\
\hline Early detection (<3 d of ECMO support) & $25^{*}$ \\
Sano/Blalock-Taussig shunt stenosis/thrombosis & 7 \\
Aortic arch stenosis/thrombosis & 6 \\
Right ventricular outflow tract/main pulmonary artery gradient & 3 \\
Branch pulmonary artery stenosis/hypoplasia & 3 \\
Pulmonary overcirculation & 2 \\
Patent ductus arteriosus & 1 \\
Pulmonary vein stenosis & 1 \\
Tricuspid regurgitation & 1 \\
Aortic valve insufficiency & 1 \\
Late detection (>3 d of ECMO support) & $13^{*}$ \\
Branch pulmonary arteries stenosis/hypoplasia & 7 \\
Pulmonary vein stenosis & 2 \\
Right ventricular outflow tract/main pulmonary artery gradient & 2 \\
Mitral regurgitation & 1 \\
Aortopulmonary collateral & 1 \\
\hline$E C M O$ Ext
\end{tabular}

$E C M O$, Extracorporeal membrane oxygenation. *More than 1 residual lesion may have been detected per patient studied. 
TABLE 3. Interventions undertaken for residual lesions detected in postoperative cardiac surgery patients placed on ECMO

\begin{tabular}{lr}
\hline \multicolumn{1}{c}{ Intervention undertaken } & $\mathbf{n}$ \\
\hline Cardiac catheterization technique & \\
Early detection ( $\leq 3$ d of ECMO duration) & 1 \\
Sano shunt dilation & 1 \\
Balloon angioplasty of branch pulmonary arteries & 1 \\
Balloon septostomy of atrial septal defect & 1 \\
Angiojet and thrombolysis of clot in distal Blalock-Taussig & \\
$\quad$ shunt and descending aorta & 2 \\
Late detection (>3 d of ECMO duration) & 1 \\
Stent placement in branch pulmonary artery & \\
Balloon dilation of atrial septal defect & 4 \\
Surgical intervention* & 3 \\
Early detection ( $\leq 3$ d of ECMO duration) & 2 \\
Sano/Blalock-Taussig shunt revision & 1 \\
Aortic arch revision & 1 \\
Branch pulmonary artery plasty & 1 \\
Right ventricular outflow tract/conduit revision & \\
Ventricular septal defect revision & 3 \\
Patent ductus arteriosus ligation & 3 \\
Late detection (>3 d of ECMO duration) & 2 \\
Branch pulmonary artery plasty & 2 \\
Blalock-Taussig shunt addition/revision & 1 \\
Right ventricular outflow tract/conduit revision & \\
Atrioventricular valve repair & \\
Pulmonary vein stenosis repair & \\
Aortopulmonary collateral ligation & \\
\hline$E C M O$, Extracorporeal membrane oxygenation. & \\
been performed per patient studied. &
\end{tabular}

bleeding from puncture sites, thrombosis of accessed vessels, or renal dysfunction. Three patients developed arrhythmias during the cardiac catheterization procedure. One patient developed complete heart block that was managed initially with a temporary pacemaker followed by permanent pacemaker placement, another patient developed atrial tachycardia that responded to adenosine in the cardiac catheterization laboratory, and the third patient developed sinus bradycardia that terminated after manipulation with the catheters ceased.

There were no significant outcome differences among patients undergoing cardiac catheterization or surgical intervention in our study population of early and late diagnosis of residual lesions. Four patients diagnosed early underwent cardiac catheterization intervention, and all of them were decannulated and survived to hospital discharge. Eleven patients diagnosed earlier had surgical intervention and 8 patients were decannulated, and 5 of them survived to hospital discharge. Three patients diagnosed later had cardiac catheterization intervention and 1 patient was decannulated successfully and survived to hospital discharge. Nine patients diagnosed later had surgical intervention and 3 of them were decannulated and 1 patient survived to hospital discharge.

The demographics, complexity of cardiac defect, and cardiac surgery and ECMO details of 35 patients with residual

lesions and 84 patients not suspected of having residual lesions are shown in Table 4. A greater number of residual lesions was detected in patients placed on ECMO for postoperative low cardiac output status with elevated serum lactate levels and high inotrope and/or high ventilator support. Twelve of 35 patients (34\%) placed on ECMO for low cardiac output status versus 13 of 84 patients $(15 \%)$ placed on ECMO for a similar indication were detected to have residual lesions $(P=.03)$.

The normalized description of our study population of 35 patients with residual lesions and 84 patients without residual lesions is shown in Figure 1. Based on ECMO support duration, 24 patients were detected as having residual lesions during the first 3 days of ECMO (early detection, group A in Figure 1), and 11 patients were detected as having residual lesions after 3 days of ECMO (late detection, group B in Figure 1). Demographic and ECMO details of

TABLE 4. Comparison of pediatric cardiac surgery patients on ECMO support detected with and without residual lesions

\begin{tabular}{|c|c|c|c|}
\hline Details & $\begin{array}{l}\text { Patients with } \\
\text { residual lesions } \\
\quad(\mathbf{n}=\mathbf{3 5})\end{array}$ & $\begin{array}{l}\text { Patients without } \\
\text { suspected } \\
\text { residual lesions } \\
(\mathbf{n}=\mathbf{8 4})\end{array}$ & $\begin{array}{c}P \\
\text { value }\end{array}$ \\
\hline Age, days; mean (IQR) & $10(5-86)$ & $13(6-72.5)$ & .74 \\
\hline Weight, kg; mean (IQR) & $3.3(2.7-4.1)$ & $3.2(2.8-4.1)$ & .99 \\
\hline \multicolumn{4}{|l|}{ Gender, n (\%) } \\
\hline Male & $15(43)$ & $37(44)$ & 1.0 \\
\hline Female & $20(57)$ & $47(56)$ & 1.0 \\
\hline \multicolumn{4}{|l|}{ Ventricle physiology, n (\%) } \\
\hline One & $20(57)$ & $38(45)$ & .31 \\
\hline Two & $15(43)$ & $46(55)$ & .31 \\
\hline \multicolumn{4}{|l|}{ RACHS, category; n (\%) } \\
\hline 2 & $4(11)$ & $7(8)$ & .73 \\
\hline 3 & $14(40)$ & $32(38)$ & .84 \\
\hline 4 & $2(6)$ & $13(16)$ & .23 \\
\hline $5-6$ & $14(40)$ & $26(31)$ & .4 \\
\hline Could not be ranked & $1(3)$ & $6(7)$ & .67 \\
\hline $\mathrm{CPB}$, min; mean (IQR) & $185(130.5-290.5)$ & $185.5(127.7-270)$ & .86 \\
\hline Xct, min; mean (IQR) & $55(30-87.5)$ & $51(27-75.2)$ & .35 \\
\hline \multicolumn{4}{|l|}{ ECMO indication, $\mathrm{n}(\%)^{*}$} \\
\hline 1 & $11(31)$ & $39(46)$ & .15 \\
\hline 2 & $12(34)$ & $13(15)$ & .03 \\
\hline 3 & $9(26)$ & $25(30)$ & .82 \\
\hline 4 & $3(9)$ & $7(8)$ & 1.0 \\
\hline $\begin{array}{l}\text { Surgery-ECMO interval, } \\
\text { days; mean (IQR) }\end{array}$ & $0(0-1)$ & $0(0-0)$ & .54 \\
\hline $\begin{array}{l}\text { ECMO duration, days; } \\
\text { mean (IQR) }\end{array}$ & $6(4.5-10.5)$ & $3(2-6)$ & $<.001$ \\
\hline Renal failure, n (\%) & $14(40)$ & $27(32)$ & .53 \\
\hline Decannulation, $\mathrm{n}(\%)$ & $25(71)$ & $50(60)$ & .3 \\
\hline Hospital discharge, $\mathrm{n}(\%)$ & $16(46)$ & $33(39)$ & .54 \\
\hline
\end{tabular}




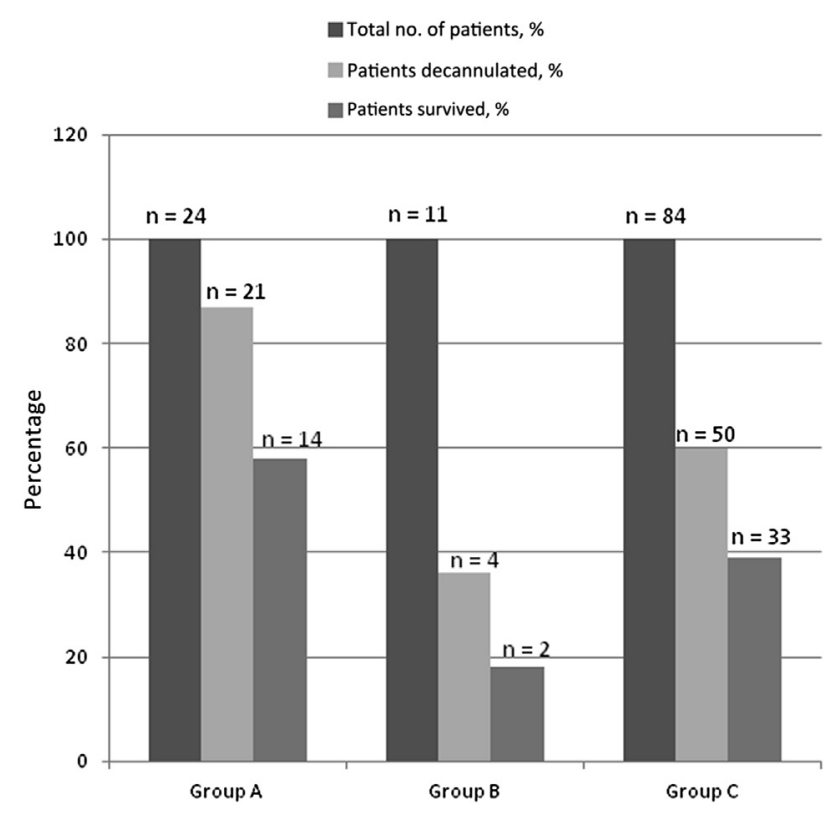

FIGURE 1. Outcome of postoperative pediatric cardiac surgery patients placed on extracorporeal membrane oxygenation (ECMO). In group A, early detection ( $\leq 3$ days of ECMO duration) of residual lesions in postoperative pediatric cardiac surgery patients placed on ECMO is noted. In group $\mathrm{B}$, late detection ( $>3$ days of ECMO duration) of residual lesions in postoperative pediatric cardiac surgery patients placed on ECMO is noted. Patients not suspected of having residual lesions are indicated in group C.

the 35 patients with residual lesions are shown in Table 5. Patients in whom residual lesions were detected later had longer cardiopulmonary bypass times $(P=.08)$ and significantly longer crossclamp times $(P=.007)$. We found that earlier detection of residual lesions was associated with significant improvement in successful decannulation from $\operatorname{ECMO}(P=.004)$ and greater survival to hospital discharge $(P=.035)$ compared with later detection (Table 5).

\section{DISCUSSION}

In our study, we found an incidence of $28 \%$ for hemodynamically significant residual lesions requiring reoperation or reintervention in postoperative pediatric cardiac surgery patients receiving ECMO support. Patients placed on ECMO support for postoperative low cardiac output status had a greater incidence of detection of residual lesions. Earlier detection of residual lesions within the first 3 days of ECMO followed by intervention improved significantly the rate of decannulation and survival to hospital discharge compared with later detection.

There has been a marked increase in ECMO use for cardiac support in children after surgical repair of congenital heart defects. ${ }^{2}$ The decision to place a child on ECMO is determined by the surgical and medical teams' judgment, and is affected by many factors, including ventricular function, magnitude of conventional inotropic support, and
TABLE 5. Comparison between patients detected to have residual lesions early ( $\leq 3$ days on ECMO) and late ( $>3$ days on ECMO)

\begin{tabular}{lccc}
\hline \multicolumn{1}{c}{ Details } & $\begin{array}{c}\text { Early detection } \\
\text { of residual } \\
\text { lesions (n=24) }\end{array}$ & $\begin{array}{c}\text { Late detection } \\
\text { of residual } \\
\text { lesion }(\mathbf{n}=\mathbf{1 1})\end{array}$ & $\begin{array}{c}\boldsymbol{P} \\
\text { value }\end{array}$ \\
\hline Age, days; mean (IQR) & $13(6.7-45)$ & $8(5-45.5)$ & .41 \\
Weight, kg; mean (IQR) & $3.3(2.7-4.2)$ & $3.3(2.6-4.1)$ & .99 \\
Ventricle physiology, n (\%) & & & \\
One & $16(67)$ & $4(36)$ & .14 \\
Two & $8(33)$ & $7(64)$ & .14 \\
CPB, min; mean (IQR) & $175.5(128-239)$ & $232(196-319)$ & .08 \\
Xct, min; mean (IQR) & $45.5(17-59)$ & $88(64.5-112)$ & .007 \\
ECMO indication, n (\%)* & & & \\
1 & $6(25)$ & $5(45)$ & .26 \\
2 & $8(33)$ & $4(37)$ & 1.0 \\
3 & $7(29)$ & $2(18)$ & .69 \\
4 & $3(13)$ & $0(0)$ & .54 \\
Surgery-ECMO interval, & $0(0-1)$ & $0(0-0)$ & .39 \\
$\quad$ days; mean (IQR) & & & \\
ECMO duration, days; & $5(3-7.3)$ & $12(8.5-13.5)$ & $<.001$ \\
mean (IQR) & & & .004 \\
Decannulation, n (\%) & $21(87)$ & $4(36)$ & .035 \\
Hospital discharge, n (\%) & $14(58)$ & $2(18)$ &
\end{tabular}

$\overline{I Q R}$, Interquartile range; $C P B$, cardiopulmonary bypass; $X c t$, crossclamp time; $E C M O$, extracorporeal membrane oxygenation. *ECMO indication: 1, off $\mathrm{CPB}$; 2, low cardiac output; 3 , cardiac arrest requiring cardiopulmonary resuscitation; 4 , other, including respiratory failure, pulmonary hypertension, and arrhythmias.

pulmonary function. The mortality rate remains high for patients supported with ECMO primarily for cardiac or hemodynamic failure, when compared with patients supported with ECMO primarily for respiratory failure. ${ }^{2}$ The high mortality in these patients is multifactorial and includes prolonged mechanical ventilation or cardiopulmonary resuscitation prior to initiation of ECMO, prolonged ECMO support, and ECMO-related complications or organ dysfunction. ${ }^{12-14}$ There are limited reports in literature that have evaluated residual lesions and their role in affecting outcomes in these patients. 5

Our study revealed residual lesions in $28 \%$ of postoperative patients placed on cardiac ECMO within 7 days of cardiac surgery. Kulik and colleagues, ${ }^{6}$ in their initial study, detected residual lesions in $22 \%$ of postoperative patients placed on ECMO. They subsequently reported residual lesions in $6 \%$ of postoperative patients. ${ }^{7}$ However, they did not place any postoperative patients requiring E-CPR on ECMO - an indication for ECMO placement that has increased steadily over a period of time. ${ }^{15}$ Chaturvedi and colleagues ${ }^{16}$ reported residual lesions in $15 \%$ of postoperative patients placed on ECMO. Their population included only patients with 2-ventricle physiology. In our study, we found a significantly greater incidence of residual lesions in patients placed on ECMO support for postoperative low cardiac output status. It is possible that, although these patients separated from cardiopulmonary bypass, they eventually developed severe myocardial dysfunction in 
the presence of hemodynamically significant residual lesions, which caused low cardiac output status and the need for ECMO support. In our population, patients who were later detected as having residual lesions had longer cardiopulmonary bypass times and significantly longer crossclamp times. We believe that the delay in detection of residual lesions in these patients is a result of an inherent tendency to allow greater time for myocardial recovery in patients with longer surgical time periods before any evaluation is undertaken.

The presence of residual lesions after pediatric cardiac surgery increases the composite morbidity-mortality outcome. ${ }^{17}$ The mortality rate is very high in pediatric patients with residual lesions receiving ECMO support after cardiac surgery in the absence of reoperation. ${ }^{9}$ Our study findings of $46 \%$ survival to hospital discharge in patients with residual lesions on ECMO support is in agreement with previously reported studies. ${ }^{5,15}$ Booth and colleagues ${ }^{5}$ reported $48 \%$ survival to hospital discharge after intervention for pediatric patients with cardiac illness placed on ECMO, and Kane and colleagues ${ }^{15}$ reported $54 \%$ survival to hospital discharge for pediatric patients with cardiac illness placed on ECMO for E-CPR. The study population in both these reports, however, included a substantial number of children with cardiac dysfunction who did not undergo cardiac surgery.

Because multiple studies have reported that longer duration of ECMO is associated with increased mortality after cardiac surgery, ${ }^{6,18,19}$ we evaluated residual lesions based on duration of ECMO support. Hintz and colleagues ${ }^{18}$ reported ECMO duration of $<3$ days to be associated significantly with improved survival in neonates placed on cardiac ECMO. They also reported a longer duration of ECMO to be the only risk factor associated with increased mortality in hypoplastic left heart syndrome patients placed on ECMO. ${ }^{18}$ Aharon and colleagues ${ }^{19}$ also reported significantly increased mortality in postoperative cardiac surgery patients placed on ECMO for $>3$ days. A longer duration of ECMO support exposes the patient to increased risks of complications, including neurologic, cardiac, renal, and pulmonary damage responsible for increased mortality. We found a significant improvement in decannulation and survival to hospital discharge in patients diagnosed with residual lesions during the first 3 days of ECMO support compared with later diagnosis and intervention. The primary goal of ECMO support during the postoperative period is to maintain tissue perfusion during myocardial recovery. Reversibility of heart dysfunction is a key determinant to successful decannulation of ECMO that may be impaired in the presence of residual lesions. In our population, earlier detection and intervention for residual lesions may have facilitated myocardial recovery, leading to a greater rate of successful decannulation and survival.
The detection of residual lesions requires high-quality imaging studies. Transthoracic echocardiography identified $20 \%$ of the residual lesions in our study. Our results corroborate those of another study that reported 4 of the 21 residual lesions $(19 \%)$ detected correctly by transthoracic echocardiography for patients on ECMO. ${ }^{5}$ Transthoracic echocardiography is an easy-to-perform bedside imaging study for patients on ECMO and has assessed ECMO cannula positions successfully. ${ }^{20}$ However, there are several limitations for application and use of transthoracic echocardiography for assessment of residual lesions on ECMO. The changes in preload on venoarterial ECMO affect assessment of ventricular ejection fraction by echocardiography. ${ }^{21} \mathrm{~Pa}$ tients with left-to-right shunting have fractional shortening of $<10 \%$ during the course of cardiac ECMO. ${ }^{22}$ Evaluation of ejection fraction by echocardiography on ECMO has been reported to be similar between nonsurvivors and survivors after 1 month of ECMO decannulation. ${ }^{23}$ The majority of residual lesions in our study were detected in pulmonary arteries, aortas, or shunts. The residual lesions seen commonly after congenital heart surgery including residual shunts, obstructed ventricular outflow pathways, and atrioventricular valve regurgitation are not detected easily by transthoracic echocardiography. ${ }^{24,25}$ Air in the chest, bandages, open sternum, endotracheal tube and ventilator tube, pacing wires, chest tubes, and many others lead to poor acoustic windows and limit access to standard views on transthoracic echocardiography. Lack of good-quality suprasternal and parasternal views limit detailed imaging of the aorta, superior vena cava, left ventricle outflow tract (subvalvar, valvar, and supravalvar portions) as well as wall motion abnormalities. The use of alternative noninvasive imaging studies including transesophageal echocardiography on ECMO is limited by probe size in small neonates, concern for upper gastrointestinal bleeding related to coagulopathy, and lack of reliability in assessing residual lesions in left ventricular outflow tracts, right ventricular outflow tracts, left pulmonary artery, and apical interventricular septum. ${ }^{26}$

Cardiac catheterization has long served as the gold standard for the anatomic and physiologic assessment of patients with congenital heart disease. There is, however, clinical reluctance toward performing invasive investigation and possible transcatheter interventions in patients during the early postoperative period. ${ }^{27}$ Ettedgui and colleagues ${ }^{28}$ and des Jardines and colleagues ${ }^{29}$ first described the feasibility of cardiac catheterization on patients supported by ECMO. Subsequently, Booth and colleagues ${ }^{5}$ reported the safety of cardiac catheterization in pediatric patients supported on cardiac ECMO. Cardiac catheterization revealed unexpected diagnostic information of clinical importance in $91 \%$ of our patients who underwent this imaging study on ECMO. The new findings on cardiac catheterization imaging were not suspected on bedside imaging in all patients, and the bedside 
examinations were inconclusive in specifying the type, location, or significance of pathologic lesions. In the study by des Jardines and colleagus, ${ }^{26}$ clinical management was altered significantly in $73 \%$ of the 15 patients on ECMO who underwent a cardiac catheterization study. Cardiac catheterization may be helpful not only for diagnostic purposes, but also for therapeutic interventions for patients on ECMO. Seven of the 27 residual lesions were managed by catheter intervention in our study without any major complications. In the study by Zahn and colleagues, ${ }^{30} 5$ of 9 postoperative patients underwent catheter intervention on ECMO. Similarly, in the study by Booth and colleagues, ${ }^{5} 12$ of the 21 postoperative patients on ECMO underwent catheter intervention. Our findings are in agreement with recent recommendations by the American Heart Association that advocate cardiac catheterization with the potential for intervention early during the postoperative period in critically ill patients on ECMO when there is a high index of suspicion for the presence of a residual structural lesion or lesions and noninvasive diagnostics fail to identify a cause for hemodynamic and/or clinical compromise (class 1, level B). ${ }^{31}$

Our study has limitations inherent to any retrospective analysis of a single center's experience. The relatively small sample size limited our ability to analyze outcomes based on surgical diagnosis and precluded regression tests to identify independent predictors. In addition, there may be unaccounted confounders that may explain outcome differences between patients with and without residual lesions, and differences in clinical outcomes based on the timing of lesion detection. The timing of the echocardiograms performed in our study was not uniform between patients and may not have been performed using standardized ECMO flows during the weaning process. Improvised echocardiographic imaging techniques and perisurgical imaging studies in a hybrid operating suite may facilitate earlier identification of residual lesions in the future. A direct pressure measurement with a needle during the ECMO weaning trial may have facilitated detection of pressure overload in the corresponding ventricle in suspected patients. Although helpful, it remains inconclusive in specifying the type, location, or significance of pressure overload. In our retrospective study analysis, 43 patients were evaluated by echocardiography and/or cardiac catheterization for residual lesions because they were unable to be weaned successfully from ECMO. Fifty-nine of the remaining 76 patients underwent echocardiography and 1 patient underwent cardiac catheterization on ECMO that did not reveal any residual lesions. More important, it is possible that, in 51 of the 84 patients who died before discharge, cardiac catheterization may have detected residual lesions had this been pursued despite clinical judgment and echocardiographic results. Last, we were unable to differentiate whether increased mortality in later detection of residual lesions was secondary to lack of complete myocardial recovery despite intervention or a result of prolonged duration of ECMO and its related complications. Detailed autopsy studies may have helped to delineate cause of death.

Despite the study limitations, we conclude that assessment of adequacy of surgical repair and the primary reason for ECMO support should begin soon after patient stabilization. Extracorporeal membrane oxygenation decannulation should not be attempted unless identification by cardiac imaging studies and repair of the residual lesion that is expected to have a hemodynamic impact on myocardial recovery take place. Echocardiography remains a powerful imaging tool; however, we must recognize its limitations during ECMO and proceed with cardiac catheterization expediently in cases in which echocardiography alone does not explain hemodynamic compromise. Early assessment and intervention for residual lesions in postoperative cardiac surgery patients receiving ECMO support improves their outcome.

\section{References}

1. Baffes TG, Fridman JL, Bicoff JP, Whitehill JL. Extracorporeal circulation for support of palliative cardiac surgery in infants. Ann Thorac Surg. 1970;4:354-63.

2. Extracorporeal Life Support Organization. Extracorporeal membrane oxygenation registry of Extracorporeal Life Support Organization. Available at: http:// www.elso.med.umich.edu. Accessed March 9, 2011.

3. Duncan BW, Hraska V, Jonas RA, Wessel DL, Del Nido PJ, Laussen PC, et al. Mechanical circulatory support in children with cardiac disease. $J$ Thorac Cardiovasc Surg. 1999;117:529-42.

4. Chauhan S, Subin S. Extracorporeal membrane oxygenation: an anaesthesiologist's perspective: part II. Clinical and technical consideration. Ann Card Anaesth. 2012;1:69-82.

5. Booth KL, Roth SJ, Perry SB, Del Nido PJ, Wessel DL, Laussen PC. Cardiac catheterization of patients supported by extracorporeal membrane oxygenation. J Am Coll Cardiol. 2002;40:1681-6.

6. Kulik TJ, Moler FW, Palmisano JM, Custer JR, Mosca RS, Bove EL, et al. Outcome- associated factors in pediatric patients treated with extracorporeal membrane oxygenator after cardiac surgery. Circulation. 1996;94:II63-8.

7. Alsoufi B, Al-Radi OO, Gruenwald C, Lean L, Williams WG, McCrindle BW, et al. Extra-corporeal life support following cardiac surgery in children: analysis of risk factors and survival in a single institution. Eur J Cardiothorac Surg. 2009; 35:1004-11.

8. Chaturvedi RR, Macrae D, Brown KL, Schindler M, Smith EC, Davis KB, et al. Cardiac ECMO for biventricular hearts after paediatric open heart surgery. Heart. 2004;90:545-51

9. Black MD, Coles JG, Williams WG, Rebeyka IM, Trusler GA, Bohn D, et al. Determinants of success in pediatric cardiac patients undergoing extracorporeal membrane oxygenation. Ann Thorac Surg. 1995;60:133-8.

10. Kolovos NS, Bratton SL, Moler FW, Bove EL, Ohye RG, Bartlett RH, et al. Outcome of pediatric patients treated with extracorporeal life support after cardiac surgery. Ann Thorac Surg. 2003;76:1435-41.

11. Jenkins KJ, Gauvreau K, Newburger JW, Spray TL, Moller JH, Iezzoni LI. Consensus-based method for risk adjustment for surgery for congenital heart disease. J Thorac Cardiovasc Surg. 2002;123:110-8.

12. Morris MC, Ittenbach RF, Godinez RI, Portnoy JD, Tabbutt S, Hanna BD, et al. Risk factors for mortality in 137 pediatric cardiac intensive care unit patients managed with extracorporeal membrane oxygenation. Crit Care Med. 2004;32:1061-9.

13. Walters HL, Hakimi M, Rice MD, Lyons JM, Whittlesey GC, Klein MD. Pediatric cardiac surgical ECMO: multivariate analysis of risk factors for hospital death. Ann Thorac Surg. 1995;60:329-37.

14. Ravishankar C, Dominguez TE, Kreutzer J, Wernovsky G, Marino BS, Godinez R, et al. Extracorporeal membrane oxygenation after stage I reconstruction for hypoplastic left heart syndrome. Pediatr Crit Care Med. 2006;7:319-26.

15. Kane DA, Thiagarajan RR, Wypij D, Scheurer MA, Fynn-Thompson F, Emani S, et al. Rapid-response extracorporeal membrane oxygenation to support cardiopulmonary resuscitation in children with cardiac disease. Circulation. 2010; 122:S241-8. 
16. Chaturvedi RR, Macrae D, Brown KL, Schindler M, Smith EC, Davis KB, et al. Cardiac ECMO for biventricular hearts after paediatric open heart surgery. Heart. 2004;90:545-51.

17. Moga MA, Manlhiot C, Marwali EM, McCrindle BW, Van Arsdell GS, Schwartz SM. Hyperglycemia after pediatric cardiac surgery: impact of age and residual lesions. Crit Care Med. 2011;39:266-72.

18. Hintz SR, Benitz WE, Colby CE, Sheehan AM, Rycus P, Van Meurs KP. Utilization and outcomes of neonatal cardiac extracorporeal life support: 1996-2000. Pediatr Crit Care Med. 2005;6:33-8.

19. Aharon AS, Drinkwater DC Jr, Churchwell KB, Quisling SV, Reddy VS, Taylor M, et al. Extracorporeal membrane oxygenation in children after repair of congenital cardiac lesions. Ann Thorac Surg. 2001;72:2095-101.

20. Thomas TH, Price R, Ramaciotti C, Thompson M, Megison S, Lemler MS. Echocardiography, not chest radiography, for evaluation of cannula placement during pediatric extracorporeal membrane oxygenation. Pediatr Crit Care Med. 2009;10: 56-9.

21. Martin GR, Short BL. Doppler echocardiographic evaluation of cardiac performance in infants on prolonged extracorporeal membrane oxygenation. Am J Cardiol. 1988;62:929-34.

22. Tanke RB, Daniëls O, van Heijst AF, van Lier H, Festen C. Cardiac dimensions during extracorporeal membrane oxygenation. Cardiol Young. 2005;15:373-8.

23. Johnson TR, Schamberger MS, Hart JC, Turrentine MW, Brown JW. After repair, atrioventricular valve regurgitation during cardiac extracorporeal membrane oxygenation predicts survival. Ann Thorac Surg. 2003;76:848-52.
24. Edwin F. eComment: outcome of extracorporeal membrane oxygenation in pediatric cardiac surgery: impact of residual lesions. Interact Cardiovasc Thorac Surg. 2010;5:758-9.

25. Soongswang J, Nana A, Laohaprasitiporn D, Durongpisitkul K, Kangkagate C Rochanasiri W, et al. Limitation of transthoracic echocardiography in the diagnosis of congenital heart diseases. J Med Assoc Thai. 2000;83:S111-7.

26. Rosenfeld HM, Gentles TL, Wernovsky G, Laussen PC, Jonas RA, Mayer JE, et al. Utility of intraoperative transesophageal echocardiography in the assessment of residual cardiac defects. Pediatr Cardiol. 1998;19:346-51.

27. Vitiello R, McCrindle BW, Nykanen D, Freedom RM, Benson LN. Complications associated with pediatric cardiac catheterization. J Am Coll Cardiol. 1998;32:1433-40.

28. Ettedgui JA, Fricker FJ, Park SC, et al. Cardiac catheterization in children on extracorporeal membrane oxygenation. Cardiol Young. 1996;6:59-61.

29. des Jardins SE, Crowley DC, Beekman RH, Lloyd TR. Utility of cardiac catheterization in pediatric cardiac patients on ECMO. Catheter Cardiovasc Interv. 1999;46:62-7.

30. Zahn EM, Dobrolet NC, Nykanen DG, Ojito J, Hannan RL, Burke RP. Interventional catheterization performed in the early postoperative period after congenital heart surgery in children. J Am Coll Cardiol. 2004;43:1264-9.

31. Feltes TF, Bacha E, Beekman RH III, Cheatham JP, Feinstein JA, Gomes AS et al. Indications for cardiac catheterization and intervention in pediatric cardiac disease: a scientific statement from the American Heart Association. Circulation. 2011;123:2607-52. 